\section{SP0031 ARE ILLNESS PERCEPTION AND COPING STYLE ASSOCIATED WITH PATIENT DELAY?}

K. Van der Elst ${ }^{1,2}$. ${ }^{1}$ Rheumatology, University Hospitals Leuven; ${ }^{2}$ Skeletal Biology and Engineering Research Centre, KU Leuven Department of Development and Regeneration, Leuven, Belgium

EULAR recommendations for rheumatoid arthritis (RA) management advocate early intensive treatment to target. Timely diagnosis and treatment initiation in RA depends on a multitude of factors, such as healthcare organisation and referral pathways. Nevertheless, individuals seem to have a large contribution themselves by delaying their first contact with a health professional after symptom onset. This pre-diagnosis period can be described as a daunting period for persons who are later diagnosed with RA, because of experiencing symptoms they do not fully understand yet. It has been shown that when confronted with particular new symptoms, persons develop or adjust certain beliefs and attitudes that determine their understanding of, and their way of dealing with these unknown symptoms. Consequently, how persons interpret (perception) and manage (coping) their initial RA-related symptoms could influence their help-seeking behaviour. The results presented in this lecture will introduce the role of illness perception and coping in the patient delay in recently diagnosed patients with early RA. The audience will learn about the role of psychosocial aspects in patients' help-seeking behaviour, and why such aspects are worthwhile to consider when aiming for a reduction in early RA treatment delay.

\section{REFERENCE:}

[1] Van der Elst $\mathrm{K}$, et al. Are illness perception and coping style associated with the delay between symptom onset and the first general practitioner consultation in early rheumatoid arthritis management? An exploratory study within the CareRA trial. Scand J Rheumatol 2016;45(3):171-8.

Disclosure of Interest: None declared

DOI: 10.1136/annrheumdis-2018-eular.7816

\section{SP0032 THE PATIENTS' PERSPECTIVE ON DELAY IN TREATMENT}

\section{S. Makri. CYPLAR, Limassol, Cyprus}

Background: Delay in early start of treatment could cause serious problems for the patient with an RMD such as unnecessary pain suffering, deformations, depression and even disability. Often patients visit Orthopaedic doctors mainly out of ignorance. Often GPs delay for various reasons, referral to the specialist Objectives: In order to achieve the goal of good quality of life, both and patient and the GP should be informed and educated so as to recognise early symptoms and seek immediate medical advice and treatment.

Methods: The use of Campaigns in the context of "Don't delay connect today "Slogan. Media appearances, with the aim of raising awareness amongst the public and patients are very useful. The use of mobile Clinique staffed by Health professionals, advising on how to recognise early symptoms, use of printed material to stress the importance of early diagnosis. Training of GPS and orthopaedics. Use of examples

Results: The patient, through education, realises the importance of early referral to a Rheumatologist for receiving personalised and effective treatment.

Conclusions: Early diagnosis and timely treatment allow the patient with RMDs to live a good quality life. Campaigning in this direction, through information and raising awareness, can empower the patient to seek help at the early stages of the disease.

Disclosure of Interest: None declared

DOI: 10.1136/annrheumdis-2018-eular.7658

\section{SP0033 DELAY IN TREATMENT FROM THE GP PERSPECTIVE}

B. Fautrel. Rheumatology, Sorbonne Université - Paris 6/Assistance publiqueHopitaux de Paris, Paris, France

Inflammatory joint disease (IJD), i.e., rheumatoid arthritis, psoriatic arthritis or spondyloarthritis, require rapid referral to rheumatologists to get optimal specialised care, since early diagnosis and early treatment are 2 key prognosis factors. To achieve this objective, education of general practioners (GP) is of dramatic importance, since they have to detect early IJD symptoms which are sometimes partial and non specific.

The lecture will identify the key elements that have to be communicated to GPs to improve their skills in detecting IJD at very steps, as well as the most important elements to make rheumatologist appointment available to them.

Disclosure of Interest: None declared

DOI: 10.1136/annrheumdis-2018-eular.7830
THURSDAY, 14 JUNE 2018

\section{Fat and fatty acids: targets for therapy?}

\section{SP0034 ADIPOSE TISSUE INFLAMMATION: ONCE FAT WAS FAT} AND THAT WAS THAT

H. Schipper ${ }^{1,2}$. ${ }^{1}$ Pediatric Cardiology, ${ }^{2}$ Laboratory of Translational Immunology, Wilhelmina Children's Hospital/University Medical Center, Utrecht, Netherlands

Until only 100.000 years ago, humans hunted smaller creatures and gathered what they could, meanwhile being hunted by larger predators. Homo sapiens showed a spectacular leap to the top of the food chain ever since. While mankind however advanced from a cavemen diet to fast food, our adipose tissue did not adapt so quickly. Here, the evolutionary origins of adipose tissue and its implications for systemic inflammatory conditions will be discussed.

A few decades ago, adipose tissue was considered a lipid sink, evolved to store energy in times of nutritional excess and release energy in times of shortage. Indeed, multicellular organisms depend on their ability to store energy to prevent starvation. Equally important though, they rely on their ability to fight infections Several lines of evidence illustrate that adipose tissue in fact embodies the amalgamation of highly conserved metabolic and immune pathways. Whereas the fat body in Drosophila melanogaster comprises adipose tissue, liver and immunological moieties in one functional unit, adipocytes in humans and other higher organisms are reminiscent of their evolutionary origins by toll-like receptor expression cytokine production and antigen-presenting capacities. From an evolutionary perspective, adipose tissue is fully equipped to respond to inflammatory stimuli, in order to fulfil the high energy demands of an acute immune response. In modern times however, the immunological capacities of adipose tissue are more of a burden. Both in nutritional overload and systemic inflammatory conditions, the inflammatory impetus of adipose tissue can have devastating consequences.

Disclosure of Interest: None declared

DOI: 10.1136/annrheumdis-2018-eular.7652

\section{SP0035 INFLAMMATION-INDUCED FORMATION OF FAT ASSOCIATED LYMPHOID CLUSTERS}

J. Caamano, on behalf of Stroma-Immune Cell Interaction Group. Institute of Immunology and Immunotherapy, College of Medical and Dental Sciences University of Birmingham, Birmingham, UK

Fat-associated lymphoid clusters (FALCs) are atypical lymphoid tissues located on adipose tissues in mucosal surfaces such as the mesenteries, omentum, mediastinum, pericardium, and gonadal fat in humans and mice. FALCs were originally identified in mouse and human mesenteries due to that they contain a high number of type 2 innate lymphoid cells (ILC). Our work has shown that FALCs contain B1, B2 and T lymphocytes as well as myeloid and other innate immune cell populations. ${ }^{1,2}$ Inflammation induces the rapid formation of FALCs and significant changes in cellular composition. Immunizations have shown the development of adaptive immune responses including B cells undergoing a germinal centre reaction in mesenteric FALCs emphasising their function on local immunity.

Our recent results show that helminth o bacterial infections induce a significant increase in proliferation of ILC in FALCs and a massive expansion in the number of clusters at early time points. A recent report has demonstrated that mesenteric FALCs act as a reservoir of CD $8+T$ resident memory cells $(\mathrm{Trm})$ that have been generated following infections with bacteria or parasites.

Our current working model is that FALCs have a dual role during immune responses. At the initial stages of infection or inflammation FALCs support the activation and proliferation of innate lymphoid cells. At later stages FALCs act as reservoirs of tissue resident memory $T$ cells through their stromal cell expression of survival factors and their association with adipocytes to support Trm metabolism and survival. Understanding what signals and cells are essential to FALC formation in homeostasis and following inflammation or infection will allow the development of therapies to reduce or prevent FALC formation during chronic inflammation and autoimmune diseases.

\section{REFERENCE:}

[1] Benezech C, et al. Nat Immunol 2015;16:819.3.

[2] Cruz-Migoni et al. Front. Immunol 2016. https://www.frontiersin.org/articles/ 10.3389/fimmu.2016.00612/full\#B27

[3] Han et al. Immunity 2017;47:1154

Disclosure of Interest: None declared DOI: 10.1136/annrheumdis-2018-eular.7848 